\title{
Gene expression profiling of lymph node metastasis by oligomicroarray analysis using laser microdissection in esophageal squamous cell carcinoma
}

\author{
YASUTO UCHIKADO ${ }^{1,2}$, HIROSHI INOUE ${ }^{2}$, NAOTSUGU HARAGUCHI ${ }^{2}$, KOSHI MIMORI $^{2}$, \\ SHOJI NATSUGOE ${ }^{1}$, HIROSHI OKUMURA ${ }^{1}$, TAKASHI AIKOU ${ }^{1}$ and MASAKI MORI ${ }^{2}$
}

\author{
${ }^{1}$ Department of Surgical Oncology, Digestive Surgery, Graduate School of Medicine, Kagoshima University, \\ 8-35-1 Sakuragaoka, Kagoshima 890-8520; ${ }^{2}$ Department of Surgery and Molecular Oncology, Medical Institute \\ of Bioregulation, Kyushu University, 4546 Tsurumihara, Beppu 874-0838, Japan
}

Received June 26, 2006; Accepted August 14, 2006

\begin{abstract}
We applied oligomicroarray analysis of 17086 genes to identify the genes related to lymph node metastasis in esophageal squamous cell carcinoma (ESCC). The samples of cancer and non-cancerous paired tissue were taken from 16 patients with ESCC who underwent esophagectomy with lymph node dissection. Total ribonucleic acid was extracted from the cancer cells obtained by using laser microdissection and was amplified by $\mathrm{T} 7$ based-amplification for the application to the oligomicroarray. The oligomicroarray demonstrated 43 overexpressed genes, such as cell-cycle regulators, cell adhesion related genes, anti-apoptosis related genes, and 138 suppressed genes such as cell differentiation related and apoptosis related genes in ESCC cells with lymph node metastasis. Among them, 5 overexpressed genes (SPP-1, CKS2, CCT5, STMN1, NDUFB9) and one suppressed expression gene $(G J B 2)$ were selected in the gene profiles, and then the expressions of those genes were confirmed by real-time semi-quantitative reverse transcriptional polymerase chain reaction (RT-PCR) method for confirmation of the result not only in study cases but also in additional 21 cases. The gene expression by real-time semi-quantitative RT-PCR was in accordance with the microarray data. Although we were able to extract some genes related to nodal metastasis in ESCC, further examination is necessary in other genes as well as the interaction of stromal tissues.
\end{abstract}

Correspondence to: Dr Masaki Mori, Department of Surgery, Medical Institute of Bioregulation, Kyushu University, Tsurumihara 4546, Beppu 874-0838, Japan

E-mail: mmori@tsurumi.beppu.kyushu-u.ac.jp

Key words: esophageal squamous cell carcinoma, lymph node metastasis, oligomicroarray, laser microdissection, gene expression profiling

\section{Introduction}

Esophageal squamous cell carcinoma (ESCC) is one of the most aggressive carcinomas of the gastrointestinal tract. Despite advances in multimodality therapy such as new surgical techniques and chemoradiotherapy, the overall 5-year survival rate in patients with ESCC still remains poor $(1,2)$. One of the more critical factors affecting ESCC patient prognosis is lymph node metastasis. Recently, there have been studies of the biological factors associated with lymph node metastasis in ESCC such as overexpression of cyclin DI (3), MMP-12 (4), $V E G F-C$ (5) and $E F-1$ (6), or reduced expression of E-cadherin (7) and cystatin $B(8)$. However, there have been only a few analyses of multiple genes expressions for the exploration mechanisms of lymph node metastasis. The development of a large scale analysis of gene expression with a microarray allows the evaluation of the gene profiles at once (9). In practice, it is important for the molecular analysis of the gene expression profile to obtain high-quality ribonucleic acid (RNA) extracted from target cells. Therefore, we introduced the laser microdissection (LMD) technique that can separate and isolate populations of cancer cells from the tumor tissue of the same patient to clearly understand the molecular changes in a tumor (10), since the tumors consist of mixed populations of carcinoma cells and stromal cells such as fibroblasts, macro-phages and lymphocytes.

In this study, combining the methods of LMD, T7-based RNA amplification and oligomicroarray, we analyzed the differentially expressed genes between the cancer cells with and without lymph node metastasis in the patients with ESCC. Furthermore, we examined and confirmed our data by real-time semi-quantitative reverse transcriptional polymerase chain reaction (RT-PCR).

\section{Patients and methods}

Patients. The samples of cancer tissues and non-cancerous tissues were obtained from 32 patients with ESCC who underwent esophagectomy with lymph node dissection between 1999 and 2003 at Kagoshima University Hospital, Kogoshima, Japan. The Ethics Committee of Kagoshima University 
Table I. The clinicopathological characteristics of the patients.

\begin{tabular}{cclccccc}
\hline No. & Age & Gender & pT & pN & No. $^{\mathrm{a}}$ & $\mathrm{pM}$ & pStage \\
\hline 1 & 55 & Male & 1 & 0 & 0 & 0 & I \\
2 & 86 & Male & 2 & 0 & 0 & 0 & IIA \\
3 & 73 & Male & 3 & 0 & 0 & 0 & IIA \\
4 & 70 & Male & 3 & 0 & 0 & 0 & IIA \\
5 & 73 & Male & 3 & 0 & 0 & 0 & IIA \\
6 & 65 & Male & 1 & 1 & 5 & $1 \mathrm{a}$ & IVA \\
7 & 70 & Male & 2 & 1 & 2 & 0 & IIB \\
8 & 76 & Male & 3 & 1 & 1 & 0 & III \\
9 & 61 & Male & 3 & 1 & 2 & 0 & III \\
10 & 74 & Male & 3 & 1 & 2 & 0 & III \\
11 & 69 & Male & 3 & 1 & 4 & 0 & III \\
12 & 69 & Male & 3 & 1 & 5 & 0 & III \\
13 & 61 & Female & 3 & 1 & 9 & $1 \mathrm{a}$ & IVA \\
14 & 58 & Male & 3 & 1 & 11 & 0 & III \\
15 & 69 & Male & 3 & 1 & 24 & $1 \mathrm{a}$ & IVA \\
16 & 68 & Male & 3 & 1 & 76 & $1 \mathrm{~b}$ & IVB \\
\hline
\end{tabular}

${ }^{\mathrm{a} N o .,}$ number of lymph node metastasis.

Hospital approved the study protocol and all patients in the study provided their written informed consent. We finally evaluated 16 of the 32 cases that passed a strict RNA quality check examination, as they were considered eligible for participation in the study. The patients ranged in age from 55 to 86 years (mean, 68.6 years). None of these patients underwent endoscopic mucosal resection, palliative resection, preoperative chemotherapy and/or radiotherapy, and none had synchronous or metachronous multiple cancer in other organs. Using the tumor node metastasis (TNM) classification of the International Union against Cancer (11), the 16 patients were classified as follows: two pT1, two pT2, twelve pT3 and none with pT4 tumors. Pathologically, all of the tumors were squamous cell carcinoma. Lymph node metastases were present in 11 of the 16 patients $(68.8 \%)$. The number of lymph node metastasis ranged from 1 to 76 in the patients who had lymph node metastasis. The M1 tumors were all due to distant lymph node metastasis (Table I).

Tissue samples and preparation of the cancer cell population by laser microdissection. All samples were immediately cut from the resected esophagus, embedded in Tissue Tek OCT medium (Sakura, Tokyo, Japan), and frozen in liquid nitrogen. The frozen tissues were sectioned by a cryostat (Leica Microsystems, Wetzlar, Germany) at $8 \mu \mathrm{m}$, mounted on glass slides and covered with PEN foil ( $2.5 \mu \mathrm{m}$ thick; Leica Microsystems). The slice samples were quickly fixed using a mixture of $100 \%$ ethanol and acetic anhydride (19:1), and stored at $-80^{\circ} \mathrm{C}$ until use. Slides were stained with hematoxylin and eosin (H\&E) at room temperature and dehydrated for $5 \mathrm{sec}$ each with 70 , 80, 95 and then $100 \%$ ethanol. After being air-dried, the sections were microdissected using the LMD system with a 337-nm nitrogen ultraviolet (UV) laser (Leica Laser Microdissection System, Leica Microsystems). The target cells dissected from a section were dropped immediately into a microcentrifuge tube cap filled with $30 \mu 1$ of RLT lysis buffer (Qiagen, Hiden, Germany). At least 600 cancer cells were collected into a $0.5-\mathrm{ml}$ tube, and then the total-RNA was extracted with an RNeasy Mini Kit (Qiagen) according to the manufacturer's instructions. The samples of paired noncancerous tissues were from at least $5 \mathrm{~cm}$ away from cancer tissue, and pathologically composed of $>75 \%$ normal esophageal squamous cells without cancerous and dysplastic cells. Total-RNA from the whole specimens of noncancerous tissue was extracted with an RNeasy Mini Kit without laser microdissection. All total-RNAs extracted from cancer cells and non-cancerous tissue were assessed for quality by electrophoretic separation on an RNA Nano LabChip or RNA 6000 Pico Labchip (Agilent Technologies, Inc., USA) in Agilent 2100 Bioanalyzer (Agilent Technologies, Inc.) according to the manufacturer's instructions.

T7-based RNA amplification and labeling. The quantities of total-RNA consisted of integrated laser microdissected cancer cells (50 ng) and non-cancerous tissues (450 ng). We carried out T7-based RNA amplification using the Low RNA Input Fluorescent Linear Amplification Kit (Agilent Technologies, Inc.). Cancer cell total-RNA was amplified for each of the 16 cases, while the non-cancerous tissues' total-RNA of the 16 cases was mixed and amplified for controllable variation in each sample. Total-RNA of all samples was reverse transcribed to first strand and second strand complementary DNA (cDNA) by MMLV-RT using an oligo(dT) primer that incorporated a T7 RNA polymerase promoter. These cDNAs were used as a template for in vitro transcription reaction in the presence of Cyanine labeled CTPs (NEW Life Science, Boston, MA) by T7 RNA polymerase. The cDNA of the non-cancerous tissue mixture was labeled with Cyanine 3-CTP (Cy3) and that of laser microdissected cancer cells was labeled with Cyanine 5-CTP (Cy5). Labeled complementary RNA (cRNA) samples were purified by using RNeasy mini kit (Qiagen). After purification, qualities of cRNA were re-evaluated on Agilent 2100 Bioanalyzer (Agilent Technologies, Inc.).

Microarray hybridization and scanning. As shown in Fig. 1, the $\mathrm{Cy} 3$, Cy5 labeled cRNA targets and control targets were mixed and hybridized with the Human 1A Oligo Microarray (Agilent Technologies, Inc.) (12). The slides were placed into an Agilent hybridization chamber (Agilent Technologies, Inc.). Hybridization proceeded at $60^{\circ} \mathrm{C}$ for $17 \mathrm{~h}$. These were then removed from the chamber and washed and dried by using the In situ Hybridization kit-plus (Agilent Technologies, Inc.) according to the Agilent array protocol. Immediate scanning of slides was performed with the Agilent dual laser DNA microarray scanner (Agilent Technologies, Inc.).

Data analysis. The intensity of each hybridization signal was evaluated using Feature Extraction software (Agilent Technologies, Inc.). The common logarithm of $\mathrm{Cy} 5 / \mathrm{Cy} 3$ ratio for each sample was calculated by averaging the spots. A cut-off value for expression level was automatically calculated according to the background fluctuation. Normalization of expression 


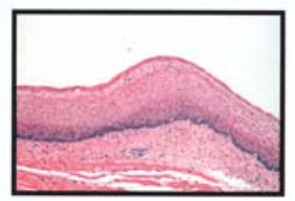

Noncancerous squamous cell tissue
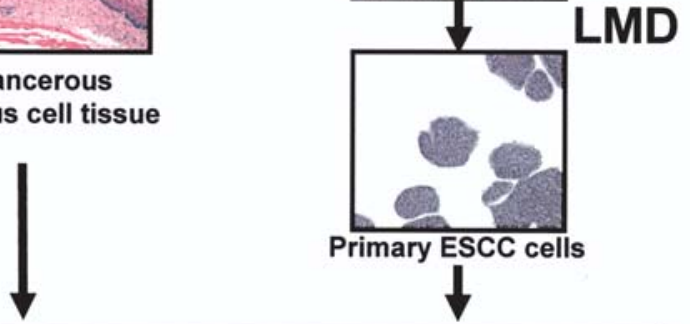

T7 linear Amplification and Labeling by Cy3/Cy5

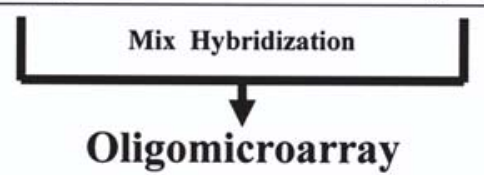

Figure 1. A schema of the LMD, T7 linear amplification and labeling by Cy3/Cy5, and oligomicroarray. The primary ESCC cells were obtained by using LMD. Whole tissue of a pair of non-cancerous squamous cells was used for the extraction of total-RNA without LMD. After the extraction of total-RNA, T7-based amplification was performed to obtain sufficient qualities of cRNA, and then the oligomicroarray analysis was performed on the noncancerous tissue and the primary esophageal carcinoma cells.

was performed using LOWESS normalization (13). Differential expression between the groups of lymph node metastasis and the group of non-lymph node metastasis was considered significant, where $\mathrm{P}<0.05$.

Real-time semi-quantitative RT-PCR. We selected 6 genes for the estimation of altered expression between lymph node positive cases and lymph node negative cases and examined their levels by applying the real-time semi-quantitative RT-PCR technique. Real-time semi-quantitative RT-PCR was carried out not only with the same samples used for array analysis but also with 21 additional samples that were not used. Firststrand cDNA synthesis was performed in a total volume of $20 \mu \mathrm{l}$ containing 300 units of M-MLV Reverse Transcriptase (Invitrogen Life Technologies, USA), $6.0 \mu 1$ of $5 \mathrm{X}$ firststrand buffer, $3.0 \mu \mathrm{l}$ of $0.1 \mathrm{M}$ DTT, $6.0 \mu \mathrm{l}$ of $10 \mathrm{mM}$ dNTP mix, $100 \mathrm{ng}$ of Random Primer (Takara Biotechnology, Japan), 20 units of RNasin Ribonucrease Inhibitor (Promega Corp., USA), 50 ng of total-RNA extracted from laser microdissected cancer cells or $450 \mathrm{ng}$ of non-cancerous tissues. Next we incubated tubes at $25^{\circ} \mathrm{C}$ for 10 min and $37^{\circ} \mathrm{C}$ for $60 \mathrm{~min}$, and then inactivated the enzyme by heating at $80^{\circ} \mathrm{C}$ for $5 \mathrm{~min}$ (14). The glyceraldehyde 3-phosphate dehydrogenase $(G A P D H)$ gene served as an internal control. The oligonucleotide primers designed for selected genes are shown in Table II. The reaction was performed in a LightCycler ${ }^{\mathrm{TM}}$ system (Roche Applied Science, Indianapolis, IN, USA) using the LightCycler-FastStart DNA Master SYBR Green I Kit (Roche Diagnostics). Each reaction was carried out in a final volume of $20 \mu \mathrm{l}$ containing $1 \mu \mathrm{l}$ of the cDNA product sample, $3 \mathrm{mM} \mathrm{MgCl}_{2}, 0.5 \mu \mathrm{M}$ of each primer and $1 \mathrm{X}$ reaction mix including FastStar DNA polymerase, reaction buffer, dNTPs and SYBR green. Thermal cycling for all genes was initiated with a denaturation step of $95^{\circ} \mathrm{C}$ for $10 \mathrm{~min}$ and consisted of
Table II. The primer sequences used in the real-time semiquantitative RT-PCR.

\begin{tabular}{|c|c|c|}
\hline $\begin{array}{l}\text { Gene } \\
\text { name }\end{array}$ & $\begin{array}{l}\text { Primer sequences } \\
\qquad\left(5^{\prime}-3^{\prime}\right)\end{array}$ & $\begin{array}{l}\text { Size } \\
\text { (bp) }\end{array}$ \\
\hline \multirow[t]{2}{*}{$S P P 1$} & TGATGGCCGAGGTGATAGTG & \multirow[t]{2}{*}{125} \\
\hline & CTCGCTTTCCATGTGTGAGG & \\
\hline \multirow[t]{2}{*}{$C K S 2$} & CTTCGACGAACACTACGAGTACC & \multirow[t]{2}{*}{112} \\
\hline & ACACCAAGTCTCСТССАСТСС & \\
\hline \multirow[t]{2}{*}{ CCT5 } & AGTTAGCCAAGAGGCGGATAAG & \multirow[t]{2}{*}{136} \\
\hline & GACTTCGGTCATAGTCTGGATGG & \\
\hline \multirow[t]{2}{*}{ STMN1 } & AGAATACACTGCCTGTCGCTTG & \multirow[t]{2}{*}{128} \\
\hline & TCTTTTGACCGAGGGCTGA & \\
\hline \multirow[t]{2}{*}{ NDUFB 9} & CACCTCCTATGAGAGATACGATTGC & \multirow[t]{2}{*}{133} \\
\hline & TCCCTCCGCAGTTTCTTCC & \\
\hline \multirow[t]{2}{*}{ GJB2 } & AGCGCAGAGACCCAAC & \multirow[t]{2}{*}{163} \\
\hline & GAGCCAGATCTTTCCAATGA & \\
\hline \multirow[t]{2}{*}{ GAPDH } & TTGGTATCGTGGAAGGACTCA & \multirow[t]{2}{*}{249} \\
\hline & TGTCATCATATTTGGCAGGTTT & \\
\hline
\end{tabular}

45 cycles with denaturation at $95^{\circ} \mathrm{C}$ for $10 \mathrm{sec}$, annealing at $60^{\circ} \mathrm{C}(S P P 1, C K S 2, G J B 2, G A P D H), 62^{\circ} \mathrm{C}(S T M N 1)$ or $64^{\circ} \mathrm{C}$ (CCT5, NDUFB9) for $10 \mathrm{sec}(S P P 1, C K S 2, G J B 2, G A P D H)$ or $5 \mathrm{sec}(S T M N 1, C C T 5, N D U F B 9)$, and elongation at $72^{\circ} \mathrm{C}$ for $5 \mathrm{sec}$. At the end of the PCR cycles, melting curve analyses and electrophoresis of the products on $2 \%$ agarose gels were done to ensure the generation of the expected PCR product. The data of selected gene expression analysis were calculated as a $\log \mathrm{T} / \mathrm{N}$ ratio: the value of target gene $=\log _{10}$ [(target gene expression of cancer cells/GAPDH expression of cancer cells)/ (target gene expression of pair non-cancerous tissue/GAPDH expression of pair non-cancerous tissue)].

Statistical analysis. The associations of lymph node metastasis with expression levels were calculated with non-parametric Kruskal-Wallis and Mann-Whitney U tests. A P-value of $<0.05$ was considered statistically significant. All statistical analyses were performed using the JMP 5 for Windows software (SAS Institute Inc., Cary, NC, USA).

\section{Results}

Quality check of total-RNA after LMD and labeled cRNA after T7-based amplification. In all samples, the target cells were cut out with at least several hundred cells per slide. Consequently, about 600-1000 cells were collected for totalRNA extraction. Total-RNA extracted after LMD had kept high quality without degradation in strict check by Agilent 2100 BioAnalyser (Fig. 2A and B). We selected 16 of 32 cases $(50 \%)$ after RNA quality was thoroughly checked. Total-RNA extracted from primary cancer cells and non-cancerous squamous cell tissue was amplified and labeled using Cy3- or Cy5-conjugated dyes. As shown in Fig. 2C and D, total-RNA from all 16 patients was successfully amplified by an estimated 200-fold by using T7 RNA polymerase. 

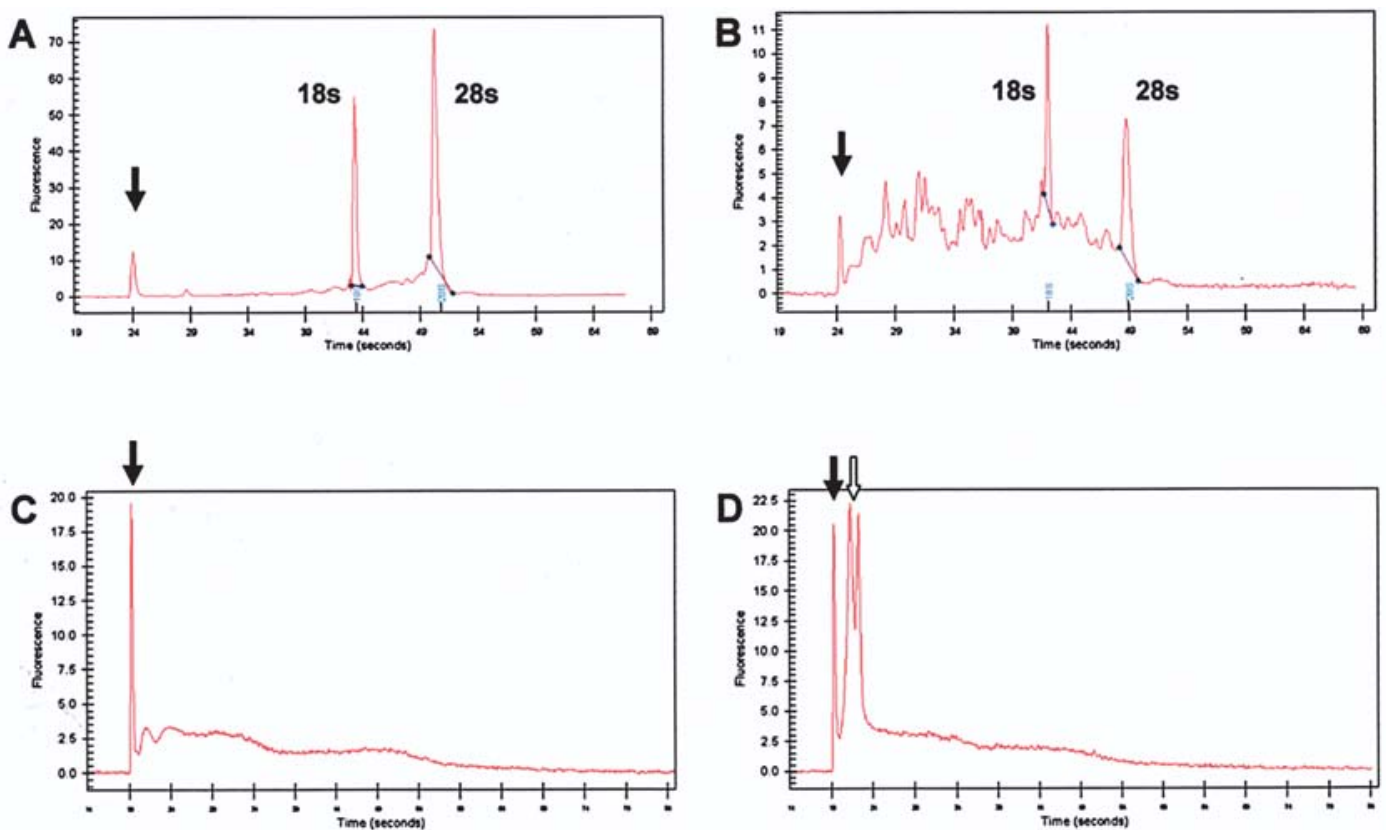

Figure 2. Quality check of total-RNA after LMD and labeled cRNA after T7-based amplification by Agilent 2100 BioAnalyser. (A), High quality total-RNA sample extracted from cancer cells with LMD. The distinct and intense features were the $18 \mathrm{~s}$ and $28 \mathrm{~s}$ ribosomal peaks, no peak between $18 \mathrm{~s}$ and $28 \mathrm{~s}$, and no peak before 18s. (B), Degraded total-RNA sample extracted from cancer cells with LMD. There was a lower $28 \mathrm{~s}$ ribosomal peak and smooth peak before $18 \mathrm{~s}$ in comparison to the high quality RNA sample. (C), The amplification and Cy3-labeling of total-RNA (450 ng/ $\mu 1)$ from non-cancerous tissue. The Cy3 labeled cRNA had the shape of a broad peak and high quality. (D), The amplification and Cy5-labeling of total-RNA (50 ng/ $\mu$ l) from primary carcinoma cells. Although the electopherograms of the Cy5 labeled cRNA had a peak of type M influenced by Cy5 (white arrow), it had the shape of a broad peak and high quality. The black arrow indicates a standard marker.

A

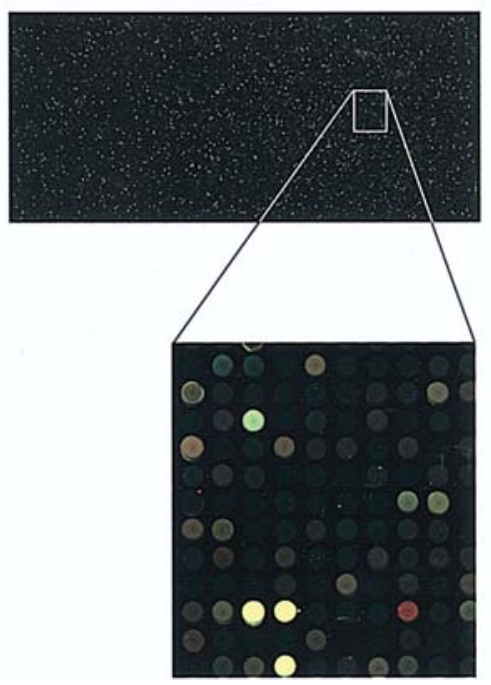

B

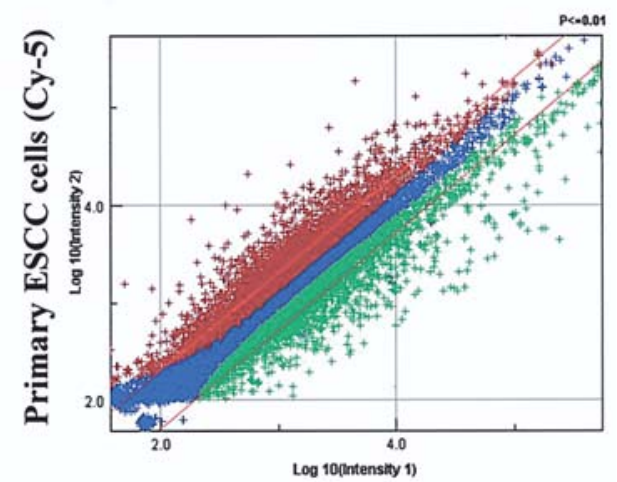

Non-cancerous tissue mix (Cy-3)

Figure 3. A representative finding of oligomicroarray expression patterns. (A), Example of the oligomicroarray data obtained from case 16 . Total 17086 genes were studied. (B), Scatter plots of oligomicroarray analysis. Primary ESCC cells (Cy5) and non-cancerous tissue mix (Cy-3) from case no. 16 were labeled and hybridized to the oligomicroarray. The red spots illustrate overexpressed genes, while the green spots were suppressed genes, and blue spots were unchanged genes.

Identification of differentially expressed genes associated with lymph node metastasis by oligomicroarray. Each cRNA probe was hybridized to oligomicroarray with 17086 genes. We evaluated the expression profiles between the cancer cells and non-cancerous squamous cell tissue (Fig. 3). A representative scatter plot of microarray analysis between the cancer tissue and non-cancerous tissue in case 16 is shown in Fig. 3B.
Overexpressed, suppressed and unchanged genes were indicated by red, green and blue spots, respectively. Each case was successfully analyzed as overexpressed, suppressed, and unchanged genes using microarray.

To identify the genes related to lymph node metastasis, 16 patients were divided into two groups: a metastatic group in which lymph node metastasis was positive in 11 patients 
Table III. Overexpressed genes correlated with lymph node metastasis.

\begin{tabular}{|c|c|c|c|}
\hline Gene name & Title & $\begin{array}{c}\text { GenBank } \\
\text { primate }\end{array}$ & $\begin{array}{l}\text { Fold } \\
\text { change }\end{array}$ \\
\hline$S P P 1$ & Secreted phosphoprotein-1 (osteopontin) & M83248.1 & 10.04 \\
\hline KRT14 & Keratin 14 & ВC042437.1 & 7.74 \\
\hline TACSTD1 & Tumor-associated calcium signal transducer 1 & AK026585.1 & 6.90 \\
\hline I_1152283 & Protein containing a GP36 envelope protein domain & AK096414.1 & 6.00 \\
\hline STMN1 & Stathmin (oncoprotein 18) & X53305.1 & 4.03 \\
\hline COL7Al & Collagen type VII alpha 1 & NM_000094.2 & 3.98 \\
\hline COLIAI & Alpha 1 subunit of type I collagen & ВС036531.1 & 3.78 \\
\hline BIRC5 & Survivin & U75285.1 & 3.65 \\
\hline$H 2 A F Z$ & $\mathrm{H} 2 \mathrm{~A}$ histone family $\mathrm{Z}$ & ВC018002.1 & 3.63 \\
\hline$C K S 2$ & Protein that binds to $\mathrm{CDC} 2 / \mathrm{CDC} 28$ protein kinase & ВС006458.1 & 3.51 \\
\hline CCT5 & Chaperonin containing T-complex 1 subunit 5 (epsilon) & ВС035499.1 & 3.40 \\
\hline I_957781 & Retired, primase polypeptide $2 \mathrm{a}$ & NM_000947.1 & 3.39 \\
\hline$M L F 1$ & Myelodysplasia/myeloid leukemia factor 1 & L49054.1 & 3.36 \\
\hline$C K S 1 B$ & CDC28 protein kinase 1 & ВC001425.1 & 3.34 \\
\hline STK31 & Homo sapiens serine/threonine kinase 31 & NM_031414.2 & 3.23 \\
\hline$T K 1$ & Thymidine kinase 1 & NM_003258.1 & 3.22 \\
\hline KIFCl & Kinesin-like 2 & ВС000712.1 & 3.07 \\
\hline CTSZ & Cathepsin Z (cathepsin X) & ВС042168.1 & 3.01 \\
\hline$C E N P F$ & Centromere protein $\mathrm{F}(350 / 400 \mathrm{kDa}$, mitosin) & U30872.1 & 3.00 \\
\hline$B G 1$ & Lipidosin & NM_015162.3 & 2.97 \\
\hline$D E F B 105$ & Homo sapiens defensin, beta 105 (DEFB105), mRNA & NM_152250.1 & 2.94 \\
\hline$M C M 7$ & Minichromosome maintenance deficient 7 & NM_005916.2 & 2.94 \\
\hline$A T P 1 B 3$ & $\mathrm{Na}^{+} / \mathrm{K}^{+}$transporting ATPase beta 3 subunit & ВC011835.1 & 2.84 \\
\hline NM_144668.1 & Homo sapiens hypothetical protein MGC33630 & NM_144668.1 & 2.77 \\
\hline$M Y N N$ & Myoneurin & ВС033620.1 & 2.77 \\
\hline$A N L N$ & Aniline & AF273437.1 & 2.74 \\
\hline I_960942 & Retired, member of the strictosidine synthase family & AB033767.1 & 2.74 \\
\hline KPNA2 & Karyopherin alpha 2 (importin alpha 1) & U09559.1 & 2.68 \\
\hline$H 2 A V$ & Protein with high similarity to S. cerevisiae Htz1p & ВС000098.1 & 2.65 \\
\hline$H M G 4 L$ & Homo sapiens high-mobility group protein 4-like & NM_178467.1 & 2.64 \\
\hline SPAG5 & Sperm associated antigen 5 & NM_006461.1 & 2.64 \\
\hline LOC 152217 & Protein of unknown function & AL832915.1 & 2.63 \\
\hline LIMS1 & LIM and senescent cell antigen-like domains 1 & ВС005341.1 & 2.51 \\
\hline ENAH & Mammalian Ena (NPC derived proline rich protein 1) & NM_018212.1 & 2.47 \\
\hline$C D C 20$ & Cell division cycle 20 & ВС000624.1 & 2.46 \\
\hline MSH6 & MutS homolog 6 (E. coli) & U28946.1 & 2.45 \\
\hline I_941570 & Protein of unknown function & ВC035925.1 & 2.45 \\
\hline HSPE1 & Chaperonin 10 & ВC023518.1 & 2.44 \\
\hline NDUFB 9 & NADH dehydrogenase ubiquinone 1 beta subcomplex 9 & AF044956.1 & 2.41 \\
\hline$S N R P G$ & Sm core protein $\mathrm{G}$ & ВC022432.1 & 2.40 \\
\hline$R P A 3$ & Replication protein A 3 & L07493.1 & 2.34 \\
\hline$A T R$ & Ataxia telangiectasia and Rad3 related & U49844.1 & 2.29 \\
\hline MGC10911 & Protein of unknown function & ВС004308.1 & 2.24 \\
\hline
\end{tabular}

(nos. 6-11) and a non-metastatic group in which lymph node metastasis was negative in 5 patients (nos. 1-5) (Table I). When comparing gene expression profiles between two groups, 43 overexpressed genes and 138 suppressed genes were significantly correlated with lymph node metastasis (Tables III and IV). The overexpressed genes such as cell cycle regulation, 
Table IV. Suppressed genes correlated with lymph node metastasis.

\begin{tabular}{|c|c|c|c|}
\hline Gene name & Title & $\begin{array}{l}\text { GenBank } \\
\text { primate }\end{array}$ & $\begin{array}{l}\text { Fold } \\
\text { change }\end{array}$ \\
\hline$M A L$ & T-lymphocyte maturation associated protein & BC003006.1 & -31.50 \\
\hline Clorf10 & Chromosome 1 open reading frame 10 & ВС030807.1 & -23.24 \\
\hline NICE-1 & NICE-1 protein & AJ243662.1 & -16.17 \\
\hline SPINK5 & Serine protease inhibitor (Kazal type) 5 & NM_006846.1 & -15.50 \\
\hline$H B B$ & Hemoglobin beta subunit & ВC007075.1 & -14.44 \\
\hline EMP1 & Echinoderm microtubule-associated protein-like & U77085.1 & -13.71 \\
\hline TGM1 & Transglutaminase 1 & ВС034699.1 & -13.47 \\
\hline$S C E L$ & Homo sapiens sciellin (SCEL) & NM_003843.2 & -13.16 \\
\hline KRT13 & Keratin 13 & ВC002661.1 & -12.22 \\
\hline CSTB & Cystatin B (stefin B) & L03558.1 & -11.92 \\
\hline$I L I R N$ & Interleukin 1 receptor antagonist & NM_173841.1 & -11.83 \\
\hline I_1110078 & Protein of unknown function & AF093250.1 & -10.98 \\
\hline$R U N X 2$ & Homo sapiens runt-related transcription factor 2 (RUNX2) & NM_004348.1 & -10.25 \\
\hline CSTA & Cystatin A (stefin A) & X05978.1 & -9.55 \\
\hline$P P L$ & Periplakin & AF013717.1 & -9.31 \\
\hline SNTA1 & Alpha 1 syntrophin & U40571.1 & -8.95 \\
\hline CLIC 3 & Chloride intracellular channel 3 & NM_004669.1 & -8.94 \\
\hline$L A G Y$ & Protein containing a homeobox domain (homeodomain) & AF454763.1 & -8.59 \\
\hline S100A9 & S100 calcium-binding protein A9 (calgranulin B) & AF086362.1 & -8.50 \\
\hline ANXA1 & Annexin I & X05908.1 & -8.45 \\
\hline SPRR3 & Small proline-rich protein 3 & NM_005416.1 & -8.37 \\
\hline I_955703 & Retired, cystatin A (stefin A) & NM_005213.2 & -8.23 \\
\hline HAT & Airway trypsin-like protease & AB002134.1 & -8.19 \\
\hline I_958335 & Member of the trypsin family of serine proteases that contains a SEA domain & AL833167.1 & -7.84 \\
\hline RHCG & Rhesus glycoprotein type $\mathrm{C}$ & AF081497.1 & -7.57 \\
\hline XRCC5 & $\mathrm{X}$-ray repair complementing defective repair in Chinese hamster cells 5 & M30938.1 & -6.90 \\
\hline$K 5 B$ & Homo sapiens keratin 5b (K5B) & NM_173352.1 & -6.74 \\
\hline SERPINBI & Monocyte neutrophil elastase inhibitor & ВС009015.1 & -6.70 \\
\hline SPRRIA & Small proline rich protein (cornifin) 1A & L05187.1 & -6.65 \\
\hline I_959634 & Protein containing eight leucine rich repeats & ВC013767.1 & -6.57 \\
\hline MYOIA & Myosin-IA (brush border myosin I) & AF105424.1 & -6.33 \\
\hline I_966519 & Protein of unknown function & I_966519 & -6.26 \\
\hline$B C E-1$ & Protein of unknown function & NM_007005.1 & -6.24 \\
\hline I_1002369 & Retired, Secreted lacrimal proline rich protein & NM_007244.1 & -6.19 \\
\hline FLJ 21511 & Protein with low similarity to $S$ & AK025164.1 & -5.93 \\
\hline CLCA4 & Chloride channel calcium-activated & AF127035.1 & -5.90 \\
\hline PPPIR3C & Protein phosphatase 1 regulatory subunit 5 & ВC012625.1 & -5.89 \\
\hline LOC 84518 & Member of the DUF614 protein of unknown function family & NM_032488.2 & -5.85 \\
\hline TRAPPCl & Homo sapiens trafficking protein particle complex 1 & NM_021210. & -5.63 \\
\hline I_1152130 & Protein containing a calponin homology $(\mathrm{CH})$ domain & AJ010306.2 & -5.60 \\
\hline I_958949 & Protein of unknown function & I_958949 & -5.54 \\
\hline KRT4 & Keratin 4 & AK056254.1 & -5.53 \\
\hline$P S C A$ & Prostate stem cell antigen & AJ297436.1 & -5.52 \\
\hline KIAA0790 & Protein containing two SAM (sterile alpha motif) domains & NM_015278.1 & -5.51 \\
\hline CD24 & CD24 antigen (small cell lung carcinoma cluster 4 antigen) & AK026603.1 & -5.31 \\
\hline I_928865 & Retired, involucrin & ВC046391.1 & -5.30 \\
\hline IBA2 & Protein with high similarity to allograft inflammatory factor 1 & AL136566.1 & -5.29 \\
\hline FLJ20626 & Protein containing three $\mathrm{C} 2 \mathrm{H} 2$ type zinc finger domains & NM_017908.1 & -5.26 \\
\hline MECP2 & Methyl CpG binding protein 2 & AF158180.1 & -5.23 \\
\hline
\end{tabular}


Table IV. Continued.

\begin{tabular}{|c|c|c|c|}
\hline Gene name & Title & $\begin{array}{c}\text { GenBank } \\
\text { primate }\end{array}$ & $\begin{array}{l}\text { Fold } \\
\text { change }\end{array}$ \\
\hline PCDH16 & Protocadherin 16 & NM_003737.1 & -5.19 \\
\hline BRP17 & Member of the metallo-beta-lactamase family & ВC002937.1 & -5.13 \\
\hline LOC 146894 & Protein containing an immunoglobulin (Ig) domain & NM_145273.1 & -5.01 \\
\hline I_1109096 & Protein containing three immunoglobulin (Ig) domains & ВC016993.1 & -4.92 \\
\hline S100A14 & S100 calcium binding protein A14 & AY007220.1 & -4.92 \\
\hline EPS8R1 & Protein containing an Src homology 3 (SH3) domain & NM_133180.1 & -4.88 \\
\hline$M G C 4171$ & Member of the glycerophosphoryl diester phosphodiesterase family & NM_024307.1 & -4.83 \\
\hline I_959851 & Retired, lymphoid blast crisis oncogene & NM_006738.2 & -4.68 \\
\hline FUT3 & Homo sapiens fucosyltransferase 3 & NM_000149.1 & -4.61 \\
\hline$D P M 2$ & Dolichol phosphate mannose synthase regulatory subunit & AF061729.1 & -4.56 \\
\hline HMOX1 & Heme oxygenase (decycling) 1 & X06985.1 & -4.53 \\
\hline TGM3 & Transglutaminase 3 & L10386.1 & -4.33 \\
\hline I_1109231 & Retired, aplysia ras homolog I & AK096600.1 & -4.28 \\
\hline FLJ25124 & Homo sapiens hypothetical protein FLJ25124, mRNA & NM_144698.2 & -4.26 \\
\hline$S H 2 D 2 A$ & $\mathrm{SH} 2$ domain protein $2 \mathrm{~A}$ & AJ000553.1 & -4.22 \\
\hline$M G C 10540$ & Protein with low similarity to S. cerevisiae Vps $25 p$ & ВC006282.1 & -4.12 \\
\hline SCEL & Sciellin & NM_144777.1 & -4.09 \\
\hline TCAP & Titin-cap (telethonin) & ВC012628.1 & -4.05 \\
\hline PIM1 & Homo sapiens pim-1 oncogene & NM_002648. & -3.99 \\
\hline OR2A4 & Member of the rhodopsin family of $G$ protein-coupled receptors & AF399598.1 & -3.97 \\
\hline ALOX12 & Arachidonate 12-lipoxygenase & M62982.1 & -3.94 \\
\hline C16orf7 & Putative $b$ subunit of ATP synthase & AB018551.1 & -3.92 \\
\hline SPRRIA & Homo sapiens small proline-rich protein $1 \mathrm{~A}, \mathrm{mRNA}$ & NM_005987. & -3.91 \\
\hline$K L K 11$ & Kallikrein 11 & AB041036.1 & -3.80 \\
\hline EPS8R2 & Protein containing an Src homology 3 (SH3) domain & AK025824.1 & -3.78 \\
\hline I_963782 & Retired, protein containing an Src homology 3 (SH3) domain & NM_022772.1 & -3.71 \\
\hline$A R S$ & Homo sapiens ARS component B (ARS), mRNA & NM_020427.2 & -3.71 \\
\hline$M G L L$ & Monoglyceride lipase & ВC006230.1 & -3.68 \\
\hline FLJ20261 & Protein with high similarity to keratin 10 (human KRT10) & NM_019016.1 & -3.65 \\
\hline FLJ35961 & Protein containing an immunoglobulin (Ig) domain & NM_152372.1 & -3.65 \\
\hline IVL & Homo sapiens involucrin (IVL), mRNA & NM_005547.1 & -3.64 \\
\hline CCNG2 & Cyclin G2 & NM_004354.1 & -3.61 \\
\hline$C R A B P 2$ & Homo sapiens cellular retinoic acid binding protein 2 & NM_001878.2 & -3.58 \\
\hline KIAA1951 & Protein containing $13 \mathrm{C} 2 \mathrm{H} 2$ type zinc finger domains & AB075831.1 & -3.58 \\
\hline I_964796 & Retired, cannabinoid receptor 2 & X74328.1 & -3.58 \\
\hline$N U C B 2$ & Nucleobindin 2 & X76732.1 & -3.57 \\
\hline$N A G K$ & $\mathrm{~N}$-acetylglucosamine kinase & ВС001029.1 & -3.51 \\
\hline ITGB4BP & Integrin beta 4 binding protein & ВC019305.1 & -3.49 \\
\hline DNASE1L3 & Deoxyribonuclease I-like 3 (DNase $\gamma$ ) & ВC015831.1 & -3.43 \\
\hline$S U L T 2 B 1$ & Hydroxysteroid sulfotransferase cytosolic 2B1 & U92315.1 & -3.41 \\
\hline ZNF185 & Zinc finger 185 & Y09538.1 & -3.41 \\
\hline GJB2 & Gap junction protein beta 2 (connexin 26) & ВС017048.1 & -3.32 \\
\hline PDLIM1 & PDZ and LIM domain 1 & ВC000915.2 & -3.31 \\
\hline$M G C 22805$ & Protein containing two ankyrin (Ank) repeats & ВC021671.1 & -3.26 \\
\hline TIMP3 & Tissue inhibitor of metalloproteinases 3 & S78453.1 & -3.20 \\
\hline DESC1 & Differentially expressed in squamous cell carcinoma 1 & AF064819.1 & -3.17 \\
\hline DKFZp547I094 & Protein of unknown function & NM_032155.1 & -3.16 \\
\hline APM2 & Protein of unknown function & ВC004471.1 & -3.15 \\
\hline FLJ14957 & Protein of unknown function & NM_032866.1 & -3.15 \\
\hline
\end{tabular}


Table IV. Continued.

\begin{tabular}{|c|c|c|c|}
\hline Gene name & Title & $\begin{array}{c}\text { GenBank } \\
\text { primate }\end{array}$ & $\begin{array}{c}\text { Fold } \\
\text { change }\end{array}$ \\
\hline$H P G D$ & Hydroxyprostaglandin dehydrogenase $15-\mathrm{NAD}$ & AK058013.1 & -3.07 \\
\hline PIG3 & Member of the zinc-containing alcohol dehydrogenase family & ВС000474.1 & -3.05 \\
\hline$S H 3 B P 1$ & Protein with high similarity to mouse Sh3bp1 & NM_018957.2 & -3.02 \\
\hline SGP28 & Specific granule protein $28 \mathrm{kDa}$ & X94323.1 & -2.99 \\
\hline$T U B B 2$ & Tubulin beta 2 & ВС019829.1 & -2.97 \\
\hline$H E B P 2$ & Chromosome 6 open reading frame 34 & AF117616.1 & -2.94 \\
\hline SIM2 & Single-minded (Drosophila) homolog 2 & NM_005069.2 & -2.93 \\
\hline EPHAl & Ephrin receptor A1 & M18391.1 & -2.92 \\
\hline PLOD3 & Procollagen-lysine 2-oxoglutarate 5-dioxygenase 3 & AF046889.1 & -2.92 \\
\hline MASP1 & Mannan-binding lectin serine protease 1 & AF284421.1 & -2.91 \\
\hline FLJ12168 & Protein containing a TBC domain & NM_024682.1 & -2.90 \\
\hline I_1860991.FL1 & Protein of unknown function (104-aa form) & X96401.1 & -2.86 \\
\hline I_959279 & Protein containing two TPR (tetratrico peptide repeat) domains & D86980.1 & -2.85 \\
\hline IFNA6 & Interferon alpha family, gene 6 & X02958.1 & -2.85 \\
\hline I_l100026 & Protein of unknown function & AF217403.1 & -2.74 \\
\hline CTSG & Cathepsin G & M16117.1 & -2.74 \\
\hline I_961562 & Protein of unknown function & AB002354.2 & -2.72 \\
\hline MGC13102 & Protein of unknown function & NM_032323.1 & -2.71 \\
\hline AIMIL & Homo sapiens absent in melanoma 1-like (AIM1L), mRNA & NM_017977.1 & -2.69 \\
\hline SLC16A4 & Solute carrier family 16 member 4 & U59185.1 & -2.66 \\
\hline$R A G D$ & Protein with high similarity to S. cerevisiae Gtr2p & ВC003088.1 & -2.66 \\
\hline DHRS1 & Member of the short-chain dehydrogenase-reductase family & AF418205.1 & -2.64 \\
\hline SERPINB2 & Plasminogen activator inhibitor 2 & Y00630.1 & -2.58 \\
\hline I_958627 & Protein with high similarity to large form lysophospholipase & ВC035836.1 & -2.55 \\
\hline FUT5 & Fucosyltransferase 5 [alpha $(1,3)$ fucosyltransferase] & $\mathrm{U} 27330.1$ & -2.53 \\
\hline FLJ32334 & Protein of unknown function & NM_144565.1 & -2.53 \\
\hline FLJ32859 & Homo sapiens hypothetical protein FLJ32859 & NM_152539.1 & -2.49 \\
\hline FKSG2 & Protein with high similarity to histamine-releasing factor & X16064.1 & -2.47 \\
\hline SLC14A2 & Solute carrier family 14 (urea transporter) member 2 & NM_007163.2 & -2.46 \\
\hline CNTFR & Ciliary neurotrophic factor receptor & ВС001492.1 & -2.45 \\
\hline ITGA9 & Integrin alpha 9 subunit & D25303.1 & -2.45 \\
\hline$A L D H 9 A 1$ & $\gamma$-aminobutyraldehyde dehydrogenase & NM_000696.2 & -2.41 \\
\hline$G P R C 5 D$ & G protein-coupled receptor family C group 5 member D & AF209923.1 & -2.36 \\
\hline CARD6 & Protein containing a caspase recruitment domain (CARD) & NM_032587.2 & -2.35 \\
\hline $\mathrm{OR} 2 \mathrm{H} 3$ & Olfactory receptor family 2 subfamily $\mathrm{H}$ member 3 & L35475.1 & -2.35 \\
\hline DNAJC5G & Homo sapiens hypothetical protein FLJ40417 & NM_173650.1 & -2.27 \\
\hline RHO6 & GTP-binding protein & ВC026356.1 & -2.24 \\
\hline IGSF8 & Immunoglobulin superfamily member 8 & AY044845.1 & -2.23 \\
\hline $\mathrm{CDH} 12$ & Cadherin 12 (Br-cadherin) & L34057.1 & -2.22 \\
\hline PTX3 & Pentaxin-related gene & ВС039733.1 & -2.21 \\
\hline
\end{tabular}

cell adhesion and growth factor and/or proteinase were candidates related to lymph node metastasis. On the other hand, apoptosis and cell differentiation were included in the suppressed genes.

The relationship between lymph node metastasis and gene expression by real-time semi-quantitative RT-PCR. To confirm the reliability of the array data, real-time semi-quantitative RT-PCR was performed in 16 pair samples without amplification of total-RNA, which was used for array analysis, and 21 additional pairs, which were not used for the array analysis. Five genes in the overexpressed gene group and one gene in suppressed gene group were selected to measure the expression levels by real-time semi-quantitative RT-PCR. These selected 


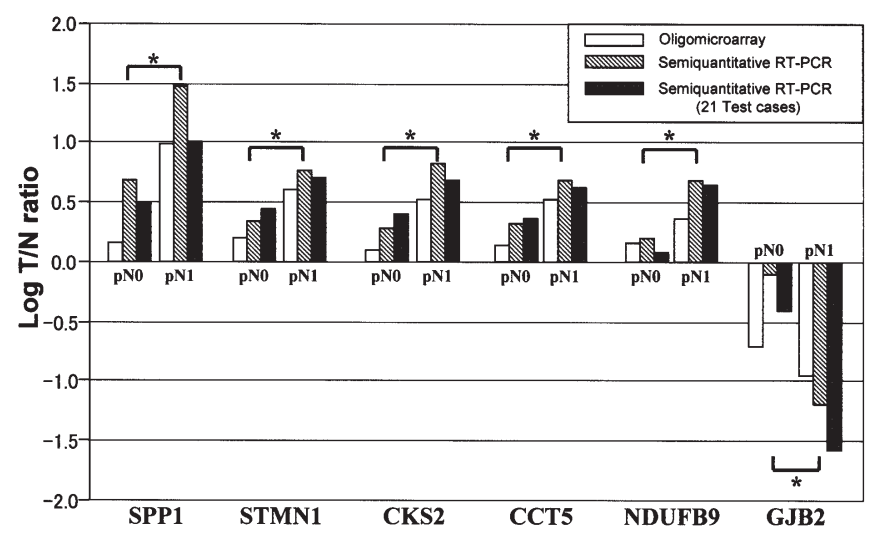

Figure 4. The relationship between lymph node metastasis and the expression of 6 genes in ESCC by real-time semi-quantitative RT-PCR. The expressions of each of the 6 genes (SPP1, STMN1, CKS2, CCT5, NDUFB9, $G J B 2$ ) was analyzed using real-time semi-quantitative RT-PCR, including 21 test cases without microarray. Significant differences were observed between the gene expressions in the cancer cells of patients with (pN1) and without (pN0) lymph node metastasis. Note the similar results between oligomicroarray analysis and analysis of real-time semi-quantitative RT$\mathrm{PCR}$, where the asterisks represent $\mathrm{P}<0.05$

genes were as follows: the overexpressed genes, SPP-1 (osteopontin), CKS2 (protein that binds to CDC2/CDC28 protein kinase), CCT5 (chaperonin containing T-complex 1 subunit 5), STMN1 (oncoprotein 18), NDUFB9 (NADH dehydrogenase ubiquinone 1 beta subcomplex 9), and suppressed gene, GJB2 (gap junction protein beta 2). The GAPDH (glyceraldehyde-3-phosphate dehydrogenase) served as an internal control.

The expression of 6 genes extracted from the 16 array samples or from the 21 additional samples was similar to the microarray data. Thus, these results support the reliability and rationality of our methodology (Fig. 4).

\section{Discussion}

Many signaling cascades controlled by multiple genes have been related to the process of carcinogenesis, tumor progression, lymph node metastasis and poor prognosis. Owing to the development of microarray technology, the correlation between clinicopathological findings and gene expression in a single experiment has been elucidated by the analysis of many gene expression profiles $(9,15)$. LMD is a powerful method to selectively extract only the desired cells from tissue specimens $(10,16)$. In this study, the analysis of the gene expression profile was performed with RNA samples obtained from 600 cancer cells by using LMD, T7amplification and oligomicroarray. It was possible to focus directly on the gene expression profile of the cancer cell population consisting of tumor tissue alone.

Lymph node metastasis is one of the most important prognostic factors in ESCC patients $(17,18)$. However, the complicated mechanisms of metastasis is not sufficient to explain the alteration of a few genes and exercise considerable influence over interstitial interaction. With this point in mind, the LMD technique enables us to analyze differential gene expressions in only cancer cells without interstitial effects. In the present study we identified gene alterations correlated with lymph node metastasis using only cancer cells. Furthermore, we successfully confirmed our data by real-time semiquantitative RT-PCR using an additional 21 test cases.

In this study, we showed that 43 overexpressed genes, including 3 unknown function genes, and 138 suppressed genes, including 12 unknown function genes, had different expression levels between the metastatic and non-metastatic groups. The overexpressed genes included cell-cycle regulators (STMN1, CKS2, MLF1, CKS1B, CDC20) (19-23), cell adhesion related genes (SPP1, TACSTD1, ENAH) (24-26), anti-apoptosis related gene (BIRC5) (27), DNA replication regulators (TK1, MCM7) $(28,29)$, and a drug resistance related gene (CCT5) (30). The suppressed genes included cell differentiation related genes (KRT4, KRT13, C1orf10, EMP1, CSTA, CSTB, S100A9, S100A14, ANXA1, SPRR1A, SPRR3, TGM3, TGM1, GJB2) (8,31-39), epithelial transporter $(R H C G)(40)$, and apoptosis related genes (MAL, DNASE1L3, PIG3) (41-43).

In these genes we selected 5 overexpressed genes and one suppressed gene, which were well documented in the literature regarding the malignant potential of some types of carcinomas. SPP-1 (osteopontin) has a role of tumor progression in breast cancer and esophageal cancer (24) and was the most overexpressed gene in the lymph node metastasis group. CKS2 (protein that binds to $C D C 2 / C D C 28$ protein kinase) is known to interact with cyclin-dependent kinases and were expressed at significantly higher levels in colon cancer (21). CCT5 (chaperonin containing T-complex 1 subunit 5) assists in the folding of actins and tubulins and showed drug resistance in gastric cancer (30), STMN1 (oncoprotein 18) acts in the regulation of the cell cycle and increased expression in acute leukemia (20), and NDUFB9 (NADH dehydrogenase ubiquinone 1 beta subcomplex 9) is a nuclear encoded mitochondrial protein with the respiratory electron transport chain $(44,45)$. GJB2 (gap junction protein beta 2 ) has a role in epidermal differentiation (39) and was suppressed in the lymph node metastasis group compared with the non-metastasis group.

These results might indicate that the important characteristics of cancer cells themselves with regards to metastatic potential to lymph nodes is the release of cell-cell adhesion, anti-apoptotic and proliferation ability. Since these genes are thought to affect each other, it is important to further analyze each gene in detail to elucidate the mechanism of lymph node metastasis in ESCC. Interestingly, we previously reported the precise function of the $M A L$ gene, the most suppressive expression gene in our data, using TE3 cells derived from lymph node metastasis of ESCC (41). When we transfected MAL cDNA into TE3 cells, those cells lost the proliferation ability and acquired apoptosis function. Since $M A L$ seemed to be one of the important candidate genes in relation to lymph node metastasis in ESCC, this gene expression should be examined by real-time semi-quantitative RT-PCR.

In conclusion, we were able to identify some genes related to lymph node metastasis in ESCC using microarray and demonstrated the close relationship between lymph node metastasis and a selection of genes extracted by gene expression profiling. In the present study, although we focused on the property of cancer cells alone, further study should be carried out on the interaction between cancer cells 
and stromal tissues. When some of the important genes related to lymph node metastasis in ESCC are clarified the genes will be useful in predicting nodal metastasis.

\section{Acknowledgements}

We thank Mayumi Oda, Harumi Yasunami, Mikiko Nagahara and Kazue Ogata for their excellent technical assistance. This study was supported by the Third Term Comprehensive Control Research for Cancer, Health and Labor Sciences Research Grants, Ministry of Health, Labor and Welfare, the Uehara Memorial Foundation, and the Sankyo Foundation of Life Science.

\section{References}

1. Mariette C, Balon JM, Piessen G, Fabre S, van Seuningen I and Triboulet JP: Pattern of recurrence following complete resection of esophageal carcinoma and factors predictive of recurrent disease. Cancer 97: 1616-1623, 2003.

2. Xiao ZF, Yang ZY, Liang J, et al: Value of radiotherapy after radical surgery for esophageal carcinoma: a report of 495 patients. Ann Thorac Surg 75: 331-336, 2003.

3. Natsugoe S, Nakashima S, Matsumoto M, et al: Biologic and imaging diagnosis of lymph node metastasis in esophageal carcinoma. J Surg Oncol 81: 25-32, 2002.

4. Ding Y, Shimada Y, Gorrin-Rivas MJ, et al: Clinicopathological significance of human macrophage metalloelastase expression in esophageal squamous cell carcinoma. Oncology 63: 378-384, 2002.

5. Kitadai Y, Amioka T, Haruma K, et al: Clinicopathological significance of vascular endothelial growth factor (VEGF)-C in human esophageal squamous cell carcinomas. Int J Cancer 93: 662-666, 2001.

6. Ogawa K, Utsunomiya T, Mimori K, et al: Clinical significance of elongation factor-1 delta mRNA expression in oesophageal carcinoma. Br J Cancer 91: 282-286, 2004.

7. Kadowaki T, Shiozaki H, Inoue M, et al: E-cadherin and alphacatenin expression in human esophageal cancer. Cancer Res 54: 291-296, 1994.

8. Shiraishi T, Mori M, Tanaka S, Sugimachi K and Akiyoshi T: Identification of cystatin B in human esophageal carcinoma, using differential displays in which the gene expression is related to lymph-node metastasis. Int J Cancer 79: 175-178, 1998.

9. Lu J, Liu Z, Xiong M, et al: Gene expression profile changes in initiation and progression of squamous cell carcinoma of esophagus. Int J Cancer 91: 288-294, 2001.

10. Mori M, Mimori K, Yoshikawa Y, et al: Analysis of the geneexpression profile regarding the progression of human gastric carcinoma. Surgery 131: S39-S47, 2002.

11. Sobin LH and Fleming ID: TNM classification of malignant tumors, 5th edition (1997). Union Internationale Contre le Cancer and the American Joint Committee on Cancer. Cancer 80: 1803-1804, 1997.

12. Van 't Veer LJ, Dai H, van de Vijver MJ, et al: Gene expression profiling predicts clinical outcome of breast cancer. Nature 415: 530-536, 2002.

13. Quackenbush J: Microarray data normalization and transformation. Nat Genet 32 (Suppl.): S496-S501, 2002.

14. Mori M, Mimori K, Inoue H, et al: Detection of cancer micrometastases in lymph nodes by reverse transcriptase-polymerase chain reaction. Cancer Res 55: 3417-3420, 1995.

15. Tamoto E, Tada M, Murakawa K, et al: Gene-expression profile changes correlated with tumor progression and lymph node metastasis in esophageal cancer. Clin Cancer Res 10: 3629-3638, 2004.

16. Kitahara O, Furukawa Y, Tanaka T, et al: Alterations of gene expression during colorectal carcinogenesis revealed by cDNA microarrays after laser-capture microdissection of tumor tissues and normal epithelia. Cancer Res 61: 3544-3549, 2001.

17. Sugimachi K, Matsuura H, Kai H, Kanematsu T, Inokuchi K and Jingu K: Prognostic factors of esophageal carcinoma: univariate and multivariate analyses. J Surg Oncol 31: 108-112, 1986.
18. Natsugoe S, Matsumoto M, Okumura H, et al: Prognostic factors in patients with submucosal esophageal cancer. J Gastrointest Surg 8: 631-635, 2004

19. Winteringham LN, Kobelke S, Williams JH, Ingley E and Klinken SP: Myeloid leukemia factor 1 inhibits erythropoietininduced differentiation, cell cycle exit and p27Kip1 accumulation. Oncogene 23: 5105-5109, 2004.

20. Melhem RF, Zhu XX, Hailat N, Strahler JR and Hanash SM: Characterization of the gene for a proliferation-related phosphoprotein (oncoprotein 18) expressed in high amounts in acute leukemia. J Biol Chem 266: 17747-17753, 1991.

21. Li M, Lin YM, Hasegawa S, et al: Genes associated with liver metastasis of colon cancer, identified by genome-wide cDNA microarray. Int J Oncol 24: 305-312, 2004.

22. Bashir T, Dorrello NV, Amador V, Guardavaccaro D and Pagano M: Control of the SCF(Skp2-Cks1) ubiquitin ligase by the APC/C(Cdh1) ubiquitin ligase. Nature 428: 190-193, 2004 .

23. Singhal S, Amin KM, Kruklitis R, et al: Alterations in cell cycle genes in early stage lung adenocarcinoma identified by expression profiling. Cancer Biol Ther 2: 291-298, 2003.

24. Tuck AB, O'Malley FP, Singhal H, Tonkin KS, Harris JF, Bautista D and Chambers AF: Osteopontin and p53 expression are associated with tumor progression in a case of synchronous, bilateral, invasive mammary carcinomas. Arch Pathol Lab Med 121: 578-584, 1997

25. Lader AS, Ramoni MF, Zetter BR, Kohane IS and Kwiatkowski DJ: Identification of a transcriptional profile associated with in vitro invasion in non-small cell lung cancer cell lines. Cancer Biol Ther 3: 624-631, 2004.

26. Di Modugno F, Bronzi G, Scanlan MJ, et al: Human Mena protein, a serex-defined antigen overexpressed in breast cancer eliciting both humoral and $\mathrm{CD} 8^{+} \mathrm{T}$-cell immune response. Int $\mathrm{J}$ Cancer 109: 909-918, 2004.

27. Blanc-Brude OP, Yu J, Simosa H, Conte MS, Sessa WC and Altieri DC: Inhibitor of apoptosis protein survivin regulates vascular injury. Nat Med 8: 987-994, 2002.

28. Wu J, Mao Y, He L, Wang N, Wu C, He Q and Skog S: A new cell proliferating marker: cytosolic thymidine kinase as compared to proliferating cell nuclear antigen in patients with colorectal carcinoma. Anticancer Res 20: 4815-4820, 2000.

29. Padmanabhan V, Callas P, Philips G, Trainer TD and Beatty BG: DNA replication regulation protein $\mathrm{Mcm} 7$ as a marker of proliferation in prostate cancer. J Clin Pathol 57: 1057-1062, 2004.

30. Ludwig A, Dietel M and Lage H: Identification of differentially expressed genes in classical and atypical multidrug-resistant gastric carcinoma cells. Anticancer Res 22: 3213-3221, 2002.

31. Marenholz I, Volz A, Ziegler A, Davies A, Ragoussis I, Korge BP and Mischke D: Genetic analysis of the epidermal differentiation complex (EDC) on human chromosome 1q21: chromosomal orientation, new markers, and a 6-Mb YAC contig. Genomics 37: 295-302, 1996.

32. Luo A, Kong J, Hu G, et al: Discovery of Ca2+-relevant and differentiation-associated genes down-regulated in esophageal squamous cell carcinoma using cDNA microarray. Oncogene 23: 1291-1299, 2004.

33. Sun W, Zhang K, Zhang X, et al: Identification of differentially expressed genes in human lung squamous cell carcinoma using suppression subtractive hybridization. Cancer Lett 212: 83-93, 2004.

34. Xu Z, Wang MR, Xu X, et al: Novel human esophagus-specific gene c1orf10: cDNA cloning, gene structure and frequent loss of expression in esophageal cancer. Genomics 69: 322-330, 2000.

35. Garcia Pedrero JM, Fernandez MP, Morgan RO, Herrero Zapatero A, Gonzalez MV, Suarez Nieto C and Rodrigo JP: Annexin A1 down-regulation in head and neck cancer is associated with epithelial differentiation status. Am J Pathol 164: 73-79, 2004.

36. Steven AC and Steinert PM: Protein composition of cornified cell envelopes of epidermal keratinocytes. J Cell Sci 107: 693-700, 1994.

37. Gonzalez HE, Gujrati M, Frederick M, et al: Identification of 9 genes differentially expressed in head and neck squamous cell carcinoma. Arch Otolaryngol Head Neck Surg 129: 754-759, 2003.

38. Yamada K, Matsuki M, Morishima Y, et al: Activation of the human transglutaminase 1 promoter in transgenic mice: terminal differentiation-specific expression of the TGM1-lacZ transgene in keratinized stratified squamous epithelia. Hum Mol Genet 6: 2223-2231, 1997. 
39. Hirschi KK, Xu CE, Tsukamoto T and Sager R: Gap junction genes Cx26 and Cx43 individually suppress the cancer phenotype of human mammary carcinoma cells and restore differentiation potential. Cell Growth Differ 7: 861-870, 1996.

40. Chen BS, Xu ZX, Xu X, et al: RhCG is down-regulated in esophageal squamous cell carcinomas, but expressed in multiple squamous epithelia. Eur J Cancer 38: 1927-1936, 2002.

41. Mimori K, Shiraishi T, Mashino K, et al: MAL gene expression in esophageal cancer suppresses motility, invasion and tumorigenicity and enhances apoptosis through the Fas pathway. Oncogene 22: 3463-3471, 2003.

42. Boulares AH and Ren T: Mechanism of acetaminophen-induced apoptosis in cultured cells: roles of caspase-3, DNA fragmentation factor, and the $\mathrm{Ca}_{2}^{+}$and $\mathrm{Mg} 2^{+}$endonuclease DNAS1L3. Basic Clin Pharmacol Toxicol 94: 19-29, 2004.
43. Robert V, Michel P, Flaman JM, et al: High frequency in esophageal cancers of p53 alterations inactivating the regulation of genes involved in cell cycle and apoptosis. Carcinogenesis 21: 563-565, 2000.

44. Lin X, Wells DE, Kimberling WJ and Kumar S: Human NDUFB9 gene: genomic organization and a possible candidate gene associated with deafness disorder mapped to chromosome 8q13. Hum Hered 49: 75-80, 1999.

45. Fang $\mathrm{N}$ and Casida JE: Anticancer action of cube insecticide: correlation for rotenoid constituents between inhibition of NADH: ubiquinone oxidoreductase and induced ornithine decarboxylase activities. Proc Natl Acad Sci USA 95: 3380-3384, 1998. 